\title{
Artificial Intelligence-Based Joint Movement Estimation Method for Football Players in Sports Training
}

\author{
Bin Zhang, ${ }^{1}$ Ming Lyu $\mathbb{D}^{\mathrm{D}}{ }^{2}$ Lei Zhang, ${ }^{1}$ and Yang $\mathrm{Wu}{ }^{1}$ \\ ${ }^{1}$ Anhui Normal University, Wuhu 241008, China \\ ${ }^{2}$ Anhui Technical College of Mechanical and Electrical Engineering, Wuhu 241002, China \\ Correspondence should be addressed to Ming Lyu; 0127000110@ahcme.edu.cn
}

Received 22 March 2021; Revised 13 April 2021; Accepted 26 April 2021; Published 4 May 2021

Academic Editor: Jianhui Lv

Copyright (c) 2021 Bin Zhang et al. This is an open access article distributed under the Creative Commons Attribution License, which permits unrestricted use, distribution, and reproduction in any medium, provided the original work is properly cited.

Football is a product in the process of human socialization; it can strengthen the body and enhance the ability of teamwork. The introduction of artificial intelligence into football training is an inevitable trend; this trend must be bound to intensify, but how to apply artificial intelligence to solve the problem of the joint movement estimation method for football players in sports training is still the main difficulty now. The basic principle of football training action pattern recognition is to determine the type of football player's action by processing and analyzing the movement information obtained by the sensor. Due to the complex movements towards football players and the changeable external environment, there are still many problems with action recognition. Focusing on the detailed classification of different sports modes, this article conducts research on the recognition of the joint movement estimation method for football players in sports training. This paper uses the recognition algorithm based on the multilayer decision tree recognizer to identify the joint movement; the experiment shows that the method used in this paper accurately identified joint movement for football players in sports training.

\section{Introduction}

Football movement pattern recognition is an emerging human-computer interaction method. According to the estimation method, football action pattern estimation can be divided into vision-based estimation technology, tactilebased estimation technology, and sensor-based estimation technology. Among these technologies, vision-based football movement estimation is to complete the classification and estimation of football movement patterns by analyzing the image or video of the active target $[1,2]$. Although the technology of this type of method is relatively mature, it has more stringent requirements for the test environment, such as good light and no objects between the moving target and the video sensor. Tactile-based movement pattern recognition limits the range of activities of the active target and requires a relatively high-cost touch screen device. Compared with the above two methods, sensor-based football joint movement estimation only requires the tester to wear a compact sensor, which has almost no limitation on the range of motion and is less disturbed by the external environment. These obvious advantages make sensor-based estimation methods gain more and more attention.

In recent years, the technology of microelectromechanical systems has become more and more mature; their size has become smaller and cheaper; their sensitivity has increased day by day. At present, micromotor systems are widely used in mobile devices, game consoles, and other products, bringing users a brand-new humancomputer interaction experience and a superior user experience. In these microelectromechanical systems, inertial measurement units (IMUs) embedded with three-axis gyroscopes and three-dimensional acceleration sensors can simultaneously obtain the angular velocity and acceleration of the measured object in the three-dimensional space, thereby providing rich and comprehensive data source. This provides a powerful data guarantee for sensor-based 
recognition and recognition of joint actions in football player training.

The main contributions to this paper are summarized as follows. (i) We use the recognition algorithm based on the multilayer decision tree recognizer to identify joint movement. (ii) We use Weka to generate the traditional SVM classifier and the traditional decision tree recognizer.

The rest of the paper is structured as follows. Section 2 introduces the Kalman algorithm based on data preprocessing. In Section 3, data feature extraction and selection is presented and analyzed. Section 4 introduces the recognition algorithm based on the single decision tree identifier and proposes the recognition algorithm based on the multilayer decision tree recognizer. The experimental results are reported in Section 5, and finally, Section 6 concludes this paper.

\section{Kalman Algorithm Based on Data Preprocessing}

2.1. Kalman Filter Algorithm. In the 1960s, Kalman first proposed the Kalman filter [3, 4], which can optimally estimate the system state based on the system state equation and a series of measurement variables with null values and containing noise, so as to achieve the purpose of noise reduction and filtering. The Kalman filter algorithm is a fast and efficient recursive estimation algorithm. Its basic calculation principle is to obtain the estimated value of the current state through the measured value of the current state and the estimated value of the previous state. The recursive estimation algorithm has a small amount of calculation, less memory, and clear steps; the Kalman filter is very suitable for real-time data processing [5] and is currently widely used in many fields such as communication, pattern recognition, navigation, and automatic control. The Kalman filter is used to estimate the state variable $x$ of a discrete-time process, which can be described by the following difference equation:

$$
x_{k}=A x_{k-1}+B u_{k-1}+w_{k-1} .
$$

Among them, $x$ is the state variable of the system, $k$ represents the system time, $x_{k}$ means the system state variable at time $k, A$ is the state transition matrix, $B$ is the gain of the system input $u$, and $w$ is the excitation noise matrix. The system observation variable is defined as $z$, and the system's measurement equation is defined as follows:

$$
z_{k}=H x_{k}+v_{k}
$$

Among them, $H$ represents the gain of the state variable against the measurement variable at time $k$ and $v$ is the observation noise matrix. Random signals $w_{k-1}$ and $v_{k}$ represent process excitation noise and observation noise, respectively; assuming that they are mutually independent, normal distribution of white noise can be obtained as follows:

$$
\begin{gathered}
p(w) \in N(0, Q), \\
p(v) \in N(0, R) .
\end{gathered}
$$

The Kalman filter algorithm has three assumptions [6, 7]: firstly, the current state is a linear function of the previous state and system input, or Gaussian noise can be superimposed; then, the measured value of the state variable must be a linear function of the state variable superimposed by Gaussian noise; finally, the initial state distribution of the system is Gaussian distribution.

Five core equations of the Kalman filter algorithm are given below. These five equations can be divided into time update equations (also called prediction functions) and measurement update equations (also called calibration functions). Among them, the time update equation calculates the a priori estimated value $x$ at the current moment (time $k$ ) according to the state estimate of the state variable $x$ at the previous moment (time $k-1$ ); the measurement update equation comprehensively calculates a priori estimated value of $x$ and the measured value of the current moment to obtain the posterior estimated value of the current moment.

Time update equations are defined as follows:

$$
\begin{aligned}
& x_{\bar{k}}=A x_{k-1}+B u_{k-1} x k-1, \\
& P_{\bar{k}}=\mathrm{AP}_{k-1} A^{T}+Q .
\end{aligned}
$$

State update equations are defined as follows:

$$
\begin{aligned}
K_{k} & =\frac{P_{\bar{k}} H^{T}}{R+\mathrm{HP}_{\bar{k}} H^{T}}, \\
x_{k} & =x_{\bar{k}}+K\left(z_{k}-H x_{\bar{k}}\right), \\
P_{k} & =\left(1-K_{k} H\right) P_{\bar{k}} .
\end{aligned}
$$

The core step of the Kalman filter algorithm is to estimate the current state of the system by combining the predicted value and the measured value: equation (4) passes the posterior estimated value $x_{k-1}$ of the previous state and the system input $u_{k-1}$ to obtain the a priori estimated value $x_{k}$ of the current state; equation (5) obtains the a priori estimation agreement variance $P_{\bar{k}}$. In equation (6), the Kalman gain matrix $K$ can be obtained by one formula, which is the intermediate calculation result of filtering. Equation (7) is used to obtain the posterior estimated value of $x_{k}$ of the current state, which is also regarded as one core step of the Kalman filter algorithm. Finally, calculate the posterior covariance $P_{k}$ of the current state for the next round of iteration.

For example, to estimate the attitude angle of a football player when running in training, the angular velocity sensor device can be used to measure and calculate in real time. But considering the influence of noise and interference on the system, the measured value is not accurate, so the real result cannot be completely dependent on the result collected by the sensor. Since any system state that satisfies the laws of physics should be continuous, the Kalman filter can predict the state at the current moment based on the estimated value of the previous moment. As for whether the result is biased toward the measured value or the estimated value, it is 
determined by $K$, the "Kalman Gain," which is calculated based on the measured value and the a priori estimated covariance.

The variables that affect the results of the Kalman filter mainly include initial state $x_{0}$, initial state covariance $P_{0}$, state transition matrix $A$, process excitation noise covariance $Q$, and measurement noise covariance $R$. Among them, the values of the initial state variable $x_{0}$ and the initial variance matrix $P_{0}$ have little effect on the filtering result because, no matter what their values are, the result can quickly converge. Therefore, this article focuses on the three factors of $A, Q$, and $R$.

2.1.1. State Transition Matrix A. The state transition matrix describes how the system transitions from the state at time $k-1$ to the state at time $k$. The system transition matrix can estimate the state of the system at the next moment. The state transition matrix may be known or unknown.

When the conversion of the system is linear, such as uniformly accelerating motion, at this time, the state transition matrix $A$ is a linear matrix. However, if the estimated process or the relationship between the observed variable and the process is nonlinear, the system does not satisfy the premise of Kalman filtering. In this case, we can consider using the Extended Kalman Filter (EKF), which uses the Jacobian matrix to linearize the expectation and variance, so the application of the Kalman filter can be extended to nonlinear systems. But EKF involves the expansion of Taylor series, so the amount of system calculations will increase.

When the system transition is uncertain, the motion equation of the moving target is usually unknown, so the state transition matrix cannot be determined. At this time, solving the problem generally requires alternative methods, such as using other prediction algorithms to replace the state transition matrix, such as the tracking Kalman filter method based on gray prediction, or using a combination of Kalman filters of multiple known models for hybrid prediction, such as the interactive multimodel method and so on.

2.1.2. Process Excitation Noise Covariance Q. The process excitation noise is the noise component superimposed by the system state variable $x$ in the state transition process. It is the white Gaussian noise with a mean value 0 and a covariance Q.

For some stable processes, it can be considered that the $Q$ value of the system is certain. At this time, it is only necessary to adjust the coefficients of the filter through experiments in order to obtain a better filtering effect. When the state transition is determined, the $Q$ value should be as small as possible, so as to ensure the faster convergence of the filter and reduce the impact on the state variables.

2.1.3. Measurement Noise Covariance R. The measurement noise covariance is a value related to the instrument and a known condition of the filter. If the value $R$ is too large or too small, it is not conducive to the final filtering effect, and the smaller the value $R$, the faster the convergence. First, calculate the appropriate $R$ value through experiments, and then, use this value to participate in the Kalman filter.

\subsection{Data Preprocessing of Joint Movement Signals for Football} Players in Sports Training. Due to the complex and changeable experimental environment, the data collected by the sensor inevitably contains missing data and noise components $[8,9]$. If these interference factors are not handled properly, the accuracy and efficiency of pattern recognition will be greatly reduced. Therefore, a series of preprocessing operations must be performed on the original data.

The data preprocessing module based on human movement signals mainly includes the following four parts: removing the null value of the original data, data normalization, Kalman filter, and data windowing.

Various movements of football players are continuous in a short period of time. To judge the joint movements of football players in training, it is necessary to judge by analyzing the data signals for a period of time, and it is necessary to perform windowing processing. Generally speaking, the duration of football player joint movement is about $2 \mathrm{~s}$. Therefore, the length of each window in this article is $2 \mathrm{~s}$. A semi-overlapping sliding window method is used to divide the data. The length of each window is 2 seconds. The data of each window processing unit is 40 .

\section{Data Feature Extraction and Selection}

The information collected by the sensor module is mainly three-axis acceleration and three-axis angular velocity data information, which can be expressed as $\vec{A}=\left(a_{x, t}, a_{y, t}, a_{z, t}\right)$ and $\vec{\omega}=\left(\omega_{x, t}, \omega_{y, t}, \omega_{z, t}\right)$, respectively, in order to reduce the complexity of multidimensional acceleration and angular velocity signal calculation, and the coordinate system of the sensor and the natural coordinate system. For the calculation between real-time conversion between the two, this paper uses the corresponding scalar, the amplitude value of the synthesized acceleration signal, and the amplitude value of the synthesized angular velocity signal; the specific calculation formula is as follows:

$$
\begin{aligned}
& A(t)=\sqrt{a_{x, t}^{2}+a_{y, t}^{2}+a_{z, t}^{2}}, \\
& \omega(t)=\sqrt{{\omega_{x, t}^{2}+\varpi_{y, t}^{2}+\varpi_{z, t}^{2}}_{x} x y z .}
\end{aligned}
$$

Among them, $a_{x, t}, a_{y, t}$, and $a_{z, t}$ represent the acceleration signal of the $x, y$, and $z$ three-axis direction changing with time; $\omega_{x, t}, \omega_{y, t}$, and $\omega_{z, t}$ represent the angular velocity signal of the $x, y$, and $z$ three-axis direction changing with time.

Covariance between angular velocity signals: the standard deviation and covariance are defined as follows:

$$
\begin{aligned}
\operatorname{std} & =\sqrt{\frac{\sum_{i=1}^{n}\left(a_{i}-a_{\mathrm{avg}}\right)^{2}}{n},} \\
\operatorname{cov}(a, \omega) & =\frac{\sum_{i=1}^{n}\left(a_{i}-a_{\mathrm{avg}}\right)\left(\omega_{i}-\omega_{\mathrm{avg}}\right)}{n-1} .
\end{aligned}
$$


Among them, $n$ represents the number of data points in an active window, $a_{i}$ and $\omega_{i}$ represent the synthetic acceleration and synthetic angular velocity at a certain time point, respectively, and $a_{\text {avg }}$ and $\omega_{\text {avg }}$, respectively, represent the average value of the synthetic acceleration and synthetic angular velocity in a sliding window.

The value of the synthetic acceleration of the football player during joint movement in training will continue to change. The point with the largest amplitude within a sliding window is the peak value. The peak value indicates the intensity of the exercise. The larger the peak value, the more intense the exercise. On the contrary, the smaller the amplitude value in a sliding window is the trough, the smaller the trough value can also indicate the stronger the signal strength. The standard deviation represents the degree of signal deviation within a period of time; the covariance represents the relationship between the change of the acceleration signal and the change of the angular velocity signal.

\section{Recognition Algorithm Based on the Decision Tree Identifier}

In recent years, pattern recognition has played an increasingly important role in the field of artificial intelligence; many important results have been achieved. Pattern recognition belongs to a kind of cognitive ability. The goal of the decision tree identifier is to supervise learning and to find a set of eigenvector in a given dataset to describe all elements; each element can be represented by a set of mutually exclusive eigenvector [10]. In other words, its goal is to distinguish a set of elements from different eigenvectors through a specific mapping relationship to form a decision tree identifier model, and this mapping relationship can be applied to unknown samples for classification.

4.1. Recognition Algorithm Based on the Single Decision Tree Identifier. The essential problem of the decision tree algorithm is to select the eigenvector of the corresponding node and the pruning of the decision tree [11]. When using the decision tree model to classify unknown samples, start from the root node and gradually identify them according to the corresponding feature attributes and further identify them according to the corresponding recognition results, until they reach the leaf nodes. The eigenvector of the decision tree algorithm is based on the value of its information gain ratio.

The purpose of the decision tree estimation method is to select the attribute with the largest information gain rate as a set of eigenvector for testing.

In order to prevent the established decision tree and training samples from overfitting and to improve the recognition accuracy and classification accuracy of unknown classes, the decision tree needs to be pruned [12]. Pruning is usually to delete the most unreliable branches through statistical methods to improve the efficiency of the entire system and the accuracy of classification.

Import 2/3 of the collected data as training samples into the Weka environment, and use the decision tree generated by the decision tree estimation C4.5, and use $1 / 3$ of the data as the sample to be tested to identify and verify its accuracy. The experimental results show that the accuracy rate has reached $95.185 \%$, but the algorithm is more complicated. There are a total of 15 leaf nodes, and many eigenvectors are used repeatedly. The generated decision tree is shown in Figure 1.

In Figure 1, A ( $\mathrm{min}$ ) represents the trough value of the composite acceleration amplitude value; A ( $\max )$ represents the peak value of the composite acceleration amplitude value; A (std) represents the standard deviation of the composite acceleration amplitude value; $\mathrm{W}(\mathrm{min})$ represents the value of the composite angular velocity amplitude value; $\mathrm{W}(\max )$ represents the peak value of the synthetic angular velocity amplitude value; W (std) represents the standard deviation of the synthetic angular velocity amplitude value; conv represents the covariance between acceleration and angular velocity.

\subsection{Recognition Algorithm Based on the Multilayer Decision} Tree Identifier. The energy consumption of the data collection terminal in football joint movement recognition is limited, so it is necessary to reduce the energy consumption of the system, while meeting the energy consumption of the system. The energy consumption of the system mainly needs to consider two aspects: sensor data collection terminal and motion recognition terminal. The energy consumed by the sensor data collection terminal mainly includes the energy consumption of the sensor collecting data, the energy consumption of sending data, and the basic energy consumption. The energy consumed by the first two factors accounts for more than $80 \%$. The energy consumed by the sensor terminal is mainly composed of three aspects: data reception, data processing, and recognition algorithms. Therefore, reducing the transmission and processing of one of the sensors' data will also reduce the system energy consumption.

Among the eight types of exercise studied in this article, we can divide them into three categories: static, slow movement, and strenuous exercise. The static movement of the football player can be distinguished from any other motion by any feature vector in the acceleration data information or the angular velocity data information. Slow movements mainly include walking, jogging, and juggling; strenuous exercise includes pass, trapping, tackle, and shoot.

Through Weka's analysis of the corresponding data, it can be obtained that slow action and violent action can be distinguished by the peak and valley value of the synthetic angular velocity and the standard deviation of the synthetic angular velocity. When the standard deviation of the synthetic angular velocity is to distinguish stationary and shooting from other actions, then the trough value of angular velocity is used to distinguish static and jogging, and the recognition rate is $98.44 \%$.

Through the analysis of football player's movements in training, it is known that the average football player spends more than $70 \%$ of the time in static and jogging state during training. If the complexity of these two movement 


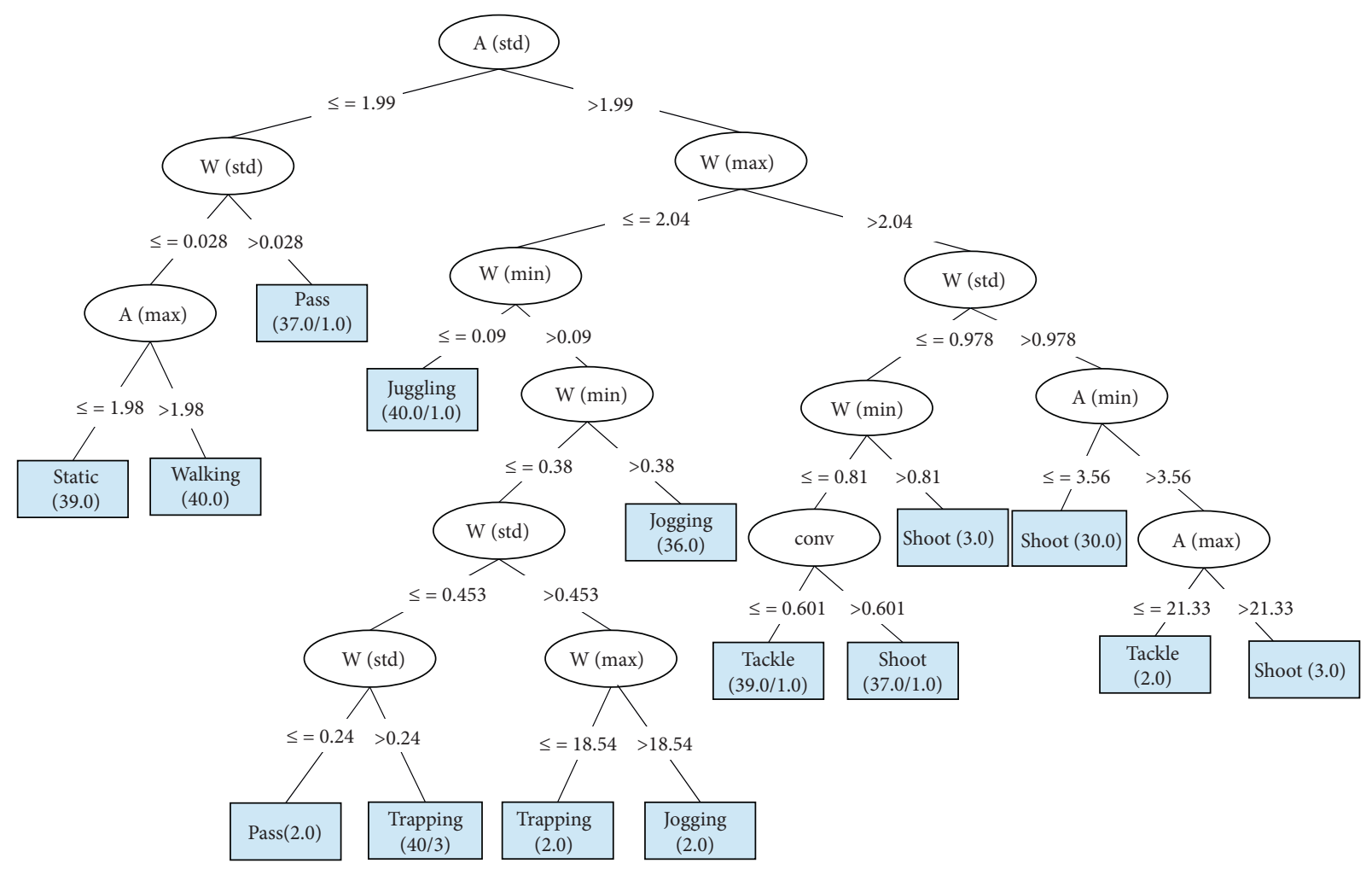

Figure 1: Recognizer based on the single decision tree.

recognition algorithms is reduced and the application of sensor data is reduced, then the energy consumption and complexity of the system will be reduced, so a recognizer algorithm based on a two-layer decision tree is proposed.

The specific implementation steps are as follows. First, extract the data of the gyroscope sensor, find the amplitude value of the synthetic angular velocity, calculate the trough value, peak value, and standard deviation of the synthetic angular velocity amplitude value, and use the first-level decision tree recognizer to identify stationary and walking, including other actions; if it is stationary and walking, the recognition is ended; otherwise, the data of the acceleration sensor is extracted; the acceleration amplitude value is calculated, and the peak value, trough value, standard deviation, and covariance of acceleration and angular velocity are obtained, and the second layer recognition is used in the device performs a second detailed identification.

4.3. First-Level Decision Tree Recognizer. In order to ensure the accuracy of the system, while reducing the energy consumption of the system, this paper hopes to achieve this by reducing the transmission of acceleration sensor data and the complexity of the system algorithm. Through experimental analysis, the common static and slow walking of the human body can be distinguished by the data characteristics of the angular velocity sensor, and the decision tree recognizer that is easy to move to the portable terminal is used for recognition. Figure 2 is a decision tree recognizer generated by Weka based on the peak value of the synthetic angular velocity, the trough value of the synthetic angular velocity, and the standard deviation of the synthetic angular velocity. Its recognition accuracy reaches $98.44 \%$.

4.4. Second-Level Decision Tree Recognizer. If the football player is not in static or walking slowly in training, it is difficult to achieve accurate recognition due to the complex movements and only relying on the time-domain characteristic signal of a single acceleration or the time-domain characteristic signal of the angular velocity. Therefore, it is necessary to further extract the data of the acceleration sensor. The feature vectors used in the second round of identification include the peak value of the synthesized acceleration signal, the bottom value of the synthesized acceleration signal, the standard deviation of the synthesized acceleration signal, and the covariance between acceleration and angular velocity. The recognizer used is still easy to implement and complicated. The decision tree recognizer has low and good real-time performance. Figure 3 shows the generation of the second-level decision tree in Weka.

In Figure 3, W (max) is the maximum value of the synthetic angular velocity, A (std) is the variance of the synthetic acceleration $[13,14], A(\max )$ is the peak value of the synthetic acceleration, A ( $\mathrm{min}$ ) is the valley value of the synthetic acceleration, and conv is the sum of the synthetic acceleration. By using some corresponding feature vectors, other actions that cannot be accurately recognized by the first-level recognizer are further recognized. Specifically, it can recognize walking, jogging, and juggling in tardiness movements and pass, trapping, tackle, and shoot in strenuous exercise. 


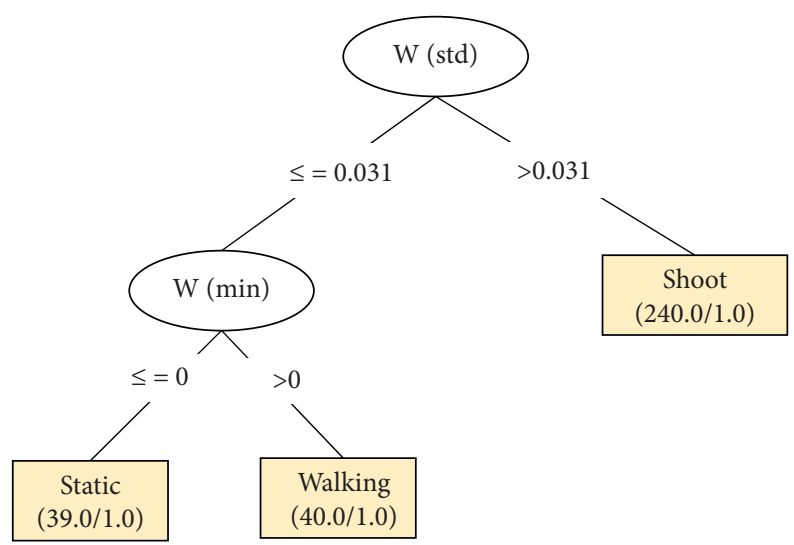

Figure 2: First-level decision tree recognizer.

Through the analysis of simulation results, the accuracy of the second-level decision tree's recognizer is $94.17 \%$. From the two decision trees generated by calculation, it can be seen that the two decision trees have 9 leaf nodes, and the common static and walking states only need to pass the first decision tree and use a gyroscope.

\section{Experiment Design and Result Analysis}

5.1. Data Extraction. In order to train an effective recognizer, a large amount of different types of joint movement data information is needed, but there is no complete database containing various football player joint movement data information. Therefore, this research needs to collect a large number of different joint movement data information of different experimenters first to train the recognizer and verify the accuracy of the recognizer [15].

The wearing position of the sensor data collection terminal will directly affect the accuracy of the recognition. For the different wearing positions of the same motion sensor, the collected sensor data will have large differences. The ideal positions for football players to wear motion sensors are feet, waist, chest, etc.

The advantage of wearing the sensor on the joints of football players' foot is that it is more sensitive to the collection of foot joint movement data and is more sensitive to some movements that use the foot; the disadvantage is that due to the high sensitivity, it will bring more interference information, stability is not very strong, and wearing comfort level is poor.

The sensor module used in this article is the MPU6050 module (including the acceleration sensor and the gyroscope sensor). Before collecting data, the scale of the sensor needs to be set. The ranges of the acceleration sensor and the gyroscope sensor are set to $\pm 16 \mathrm{~g}$ and $\pm 2000 / \mathrm{sec}$, and the frequency is $20 \mathrm{~Hz}$.

5.2. Realization of Joint Movement Recognition Algorithm and Analysis of Results. After collecting a large amount of joint movement data, it is necessary to mine and process the corresponding data. In order to process the data more professionally, this research adopts the special data processing and mining software called Weka [16].

5.2.1. Weka. In order to make better use of the collected sensor data, we use the Waikato Environment for Knowledge Analysis (Waikato Environment for Knowledge Analysis, Weka) software to process and analyze the data. Weka is an open source and free data mining platform based on the JAVA environment. It is a collection of a large number of data mining machine learning methods. This includes a large number of data preprocessing, classification, regression, clustering, association, and other rules.

The main interface of Weka mainly includes six parts, and the six parts also correspond to the corresponding functional modules:

(1) Data preprocessing: deal with the noise of the data accordingly

(2) Classify: training and testing-related classification methods

(3) Cluster: learn clustering from the trained data

(4) Correlation: learn related association rules from training data

(5) Feature selection: select data-related attributes

(6) Visualization: view the association rules of related data

In order to compare the validity and reliability of the recognition algorithm based on the two-layer decision tree recognizer proposed in this paper, we use Weka to generate the traditional SVM classifier [17] and the traditional decision tree recognizer to analyze each recognition performance.

5.2.2. Bayes Classifier. According to the principle of Bayes classifier implementation, this paper uses $2 / 3$ of the collected sensor data to train the Bayes recognizer [18, 19], and the remaining $1 / 3$ of the sensor data is used to verify the accuracy and effect of the generated recognizer. The recognition rate of the Bayesian classifier recognizer is $93.44 \%$, but 


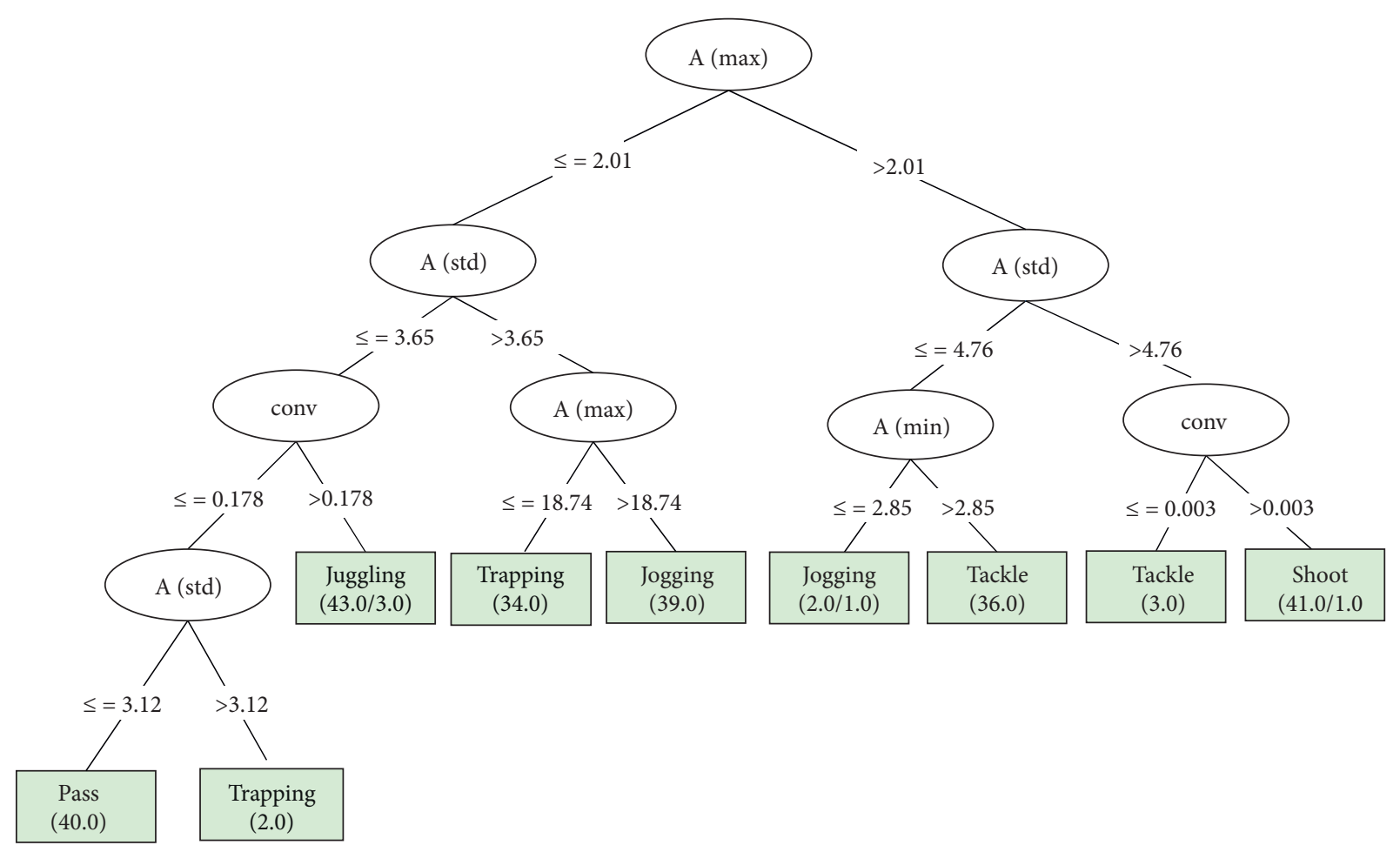

FIgURE 3: Second-level decision tree recognizer.

its algorithm is more complicated. Among them, the recognition rate of strenuous pass, trapping, tackle, and shoot is relatively low.

5.2.3. Support Vector Machine Classifier. According to the principle of the recognizer of the support vector machine, $2 / 3$ of the sensor data collected in this study is used to train the recognizer of the support vector machine, and the remaining $1 / 3$ of the sensor data is used to test the accuracy and effect of the generated recognizer. Due to the limited eigenvectors selected by the system and the limited training samples, its recognition accuracy is only $89.38 \%$, which is low for some more complex movements.

5.2.4. Compare the Effects of Different Recognizers. The traditional decision tree recognizer and the two-layer decision tree recognizer have been introduced in detail above. The coefficient of the weighted average is the weighted average of the coefficients of each category, and the weight is the proportion of the actual category. By comparing the coefficients of the classifiers, it can be found that the recognition effect based on the two-layer decision tree recognizer is relatively ideal, and it is better than a single simple Bayesian network and support vector machine recognizer; from the complexity of the algorithm and the training time, it can be seen that the former is also far superior to the latter; combined with football players' exercise habits and algorithms, the former needs to collect less than the latter two, so its system energy consumption will be reduced accordingly.

5.3. Results and Discussion. The research objects used in this paper are 20 young male football players, 2 players in a group, divided into 10 groups, aged between 18 and 26, with heights ranging from $155 \mathrm{~cm}$ to $180 \mathrm{~cm}$.

Method: the method of collecting the training set is the same as the method mentioned above. It simulates eight actions of a football player static, walking, jogging, juggling, pass, trapping, tackle, and shoot. Each action needs to be repeated five times to upload the data to the PC. As seen from Table 1, the recognition rate is above 95\%.

The verification process is to redo these eight actions on the 20 experimenters to see if there are any false positives.

Compared with other recognizers, the recognition rate of the recognition algorithm based on the multilayer decision tree recognizer proposed in this paper is higher than others, as shown in Figure 4.

From experimental results, it can be seen that the false alarm rate (meaning that one action is understood as another action) is low; the highest false alarm rate is the most vigorous tackles and shots, and the false alarms can be turned off directly. Therefore, the system in this study should also follow this principle. In general, the joint movement recognition algorithm for football players in training studied in this paper meets the goals and requirements of the system. 
TABLE 1: Recognition rate of joint movement.

\begin{tabular}{lcccccccccc}
\hline Group no. & 1 & 2 & 3 & 4 & 5 & 6 & 7 & 8 & 9 \\
\hline Correct identify & 78 & 79 & 79 & 80 & 80 & 80 & 77 & 80 & 79 \\
Unrecognized & 0 & 0 & 1 & 0 & 0 & 0 & 1 & 0 & 0 \\
False positive & 2 & 1 & 0 & 0 & 0 & 0 & 2 & 0 & 1 \\
Recognition rate (\%) & 98 & 99 & 99 & 100 & 100 & 100 & 96 & 100 & 99 & 100 \\
\hline
\end{tabular}

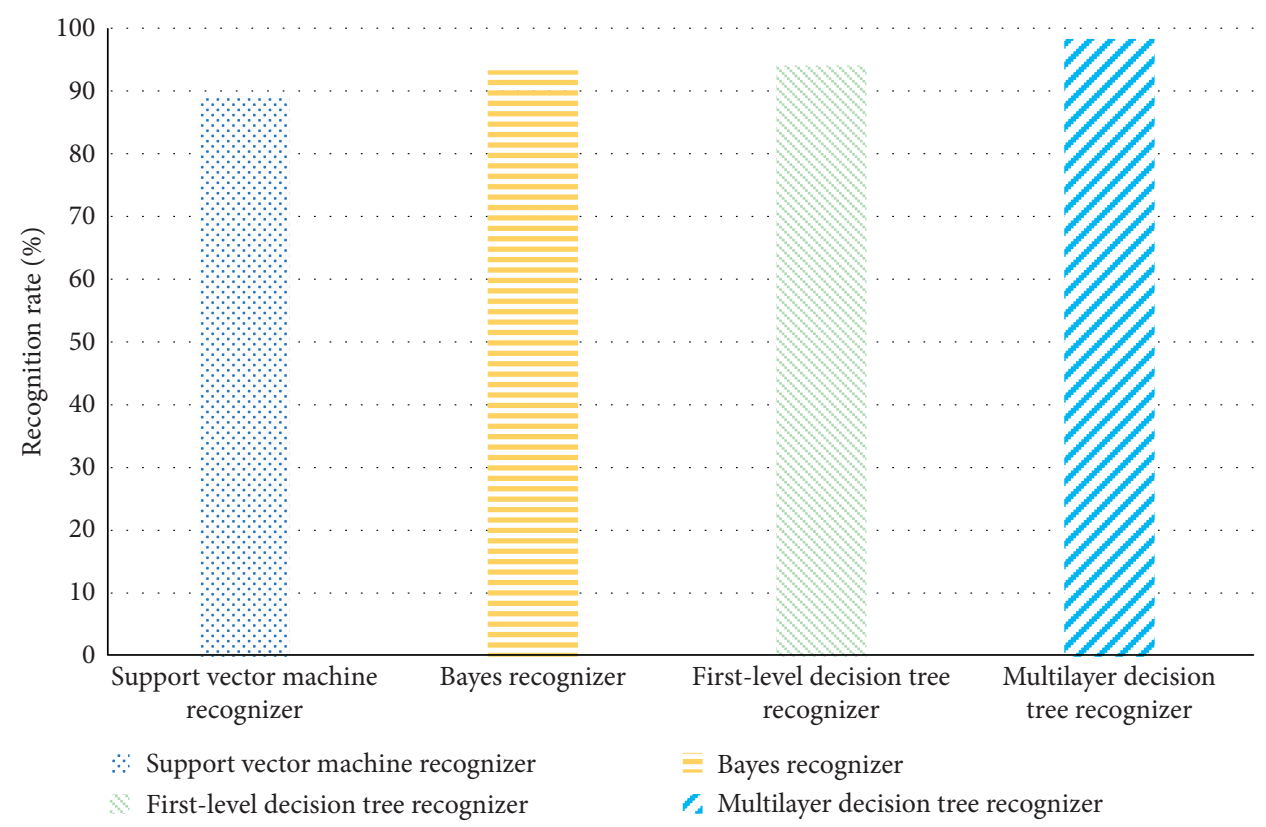

Figure 4: Recognition rate of different recognizers.

\section{Conclusions}

This paper makes an in-depth analysis of the existing pattern recognition system, analyzes the joint movement data of football players in training, and proposes a recognition algorithm based on a multilayer decision tree recognizer. Extract the data firstly, find the amplitude value of the synthetic angular velocity, calculate the peak value, trough value, and standard deviation of the synthetic angular velocity amplitude value, and use the first-level decision tree recognizer to identify stationary, walking, and also other actions; if it is stationary and walking, the recognition is ended; otherwise, the data of the acceleration sensor is extracted, the acceleration amplitude value is calculated, and the trough value, peak value, standard deviation, and covariance of acceleration and angular velocity are obtained, and the second layer recognition is used in the device performs a second detailed identification through experiments to verify that the system can meet the needs of joint movement estimation for football players in training. In this paper, the sensor module is used to collect the data information of the acceleration sensor and angular velocity sensor during the eight kinds of sports of different football players and analyze the joint movement by extracting the corresponding feature vector. In future, we will also consider various complex joint movements to better assist in sports training.

\section{Data Availability}

The data used to support the findings of this study are available from the corresponding author upon request.

\section{Conflicts of Interest}

The authors declare that they have no conflicts of interest.

\section{References}

[1] X. Jiao, "Research on key technologies of intrusion detection based on pattern recognition," in Proceedings of the 2019 7th International Conference on Machinery, Materials and Computing Technology, pp. 390-393, Aizu-Wakamatsu, Japan, March 2019.

[2] Z. Fan, S. Yu, and L. Wei, "Object recognition algorithm based on an improved convolutional neural network," Journal Beijing Institute of Technology, vol. 29, no. 2, pp. 139-145, 2020.

[3] G. Shi-Luo, Y.-J. Sun, L.-M. Chang et al., "Robust Cubature Kalman Filter Method for the Nonlinear Alignment of SINS," Defence Technology, vol. 17, no. 2, pp. 593-598, 2021.

[4] X. Sun, C. Wen, and T. Wen, "A novel step-by-step high-order extended kalman filter design for a class of complex systems with multiple," Basic Multipliers, vol. 30, no. 2, pp. 313-321, 2021. 
[5] L. Ying, J. Wang, and H. Zhongzhi, "Kalman-filter-group based aero-engine sensors fault diagnosis and verification," in Proceedings of the 2019 Chinese Intelligent Systems Conference, pp. 100-106, Singapore, September 2019.

[6] X. Chenhui, H. Defeng, and S. Xiulan, "Data attack detection of connected vehicles based on adaptive kalman filter," pp. 1-6, 2019.

[7] L. Ying, C. Lei, and Z. Zhang, "Research and simulation on pilot configuration in multi-antenna system based on kalman filter," in Proceedings of the International Conference on Mechanical Design (ICMD), pp. 371-379, Huzhou, China, August 2019.

[8] S.-Y. Hwang, L. Jang-Hyun, H. Kyu-Tack et al., "Time-series data analysis by pattern recognition and classification for fault diagnosis of plant equipment," in World Maritime Technology Conference 2018 (WMTC18), pp. 1-7, Shangai, China, December 2018.

[9] D. Ruijuan and C. Shilin, "The pattern recognition application research in image processing," in 2018 5th International Conference on Electrical \& Electronics Engineering and Computer Science (ICEEECS 2018), pp. 214-219, Istanbul, Turkey, May 2018.

[10] P. Oleksiy, I. Hernandez-Bautista, N. Oscar Camacho et al., "Wavelet filter adjusting for image lossless compression using pattern recognition," in Proceedings of the Mexican Conference on Pattern Recognition, pp. 221-230, Springer, Cancun, Mexico, June 2014.

[11] W. Chen, "Research on group decision making of large-scale engineering based on uncertain decision tree classification algorithms," in Proceedings of the 2019 4th International Industrial Informatics and Computer Engineering Conference (IIICEC 2019), pp. 383-387, Sanya, China, December 2019.

[12] Y. Huizhen, C. Xiaohang, Z. Wang et al., "Research on material identification technology of sealed relay remainder material based on decision tree," in Proceedings of the 7th International Conference on Reliability of Electrical Products and Electrical Contacts, pp. 237-243, Suzhou, China, November 2019.

[13] K. Yong-Joong and C. Sung-Bae, "A HMM-based location prediction framework with location recognizer combining k-nearest neighbor and multiple decision trees," in Proceedings of the International Conference on Hybrid Artificial Intelligence Systems, pp. 618-628, Salamanca, Spain, September 2013.

[14] K. Kyoung Min, P. Joon Jo, S. Myung Hyun et al., "Binary decision tree using K-means and genetic algorithm for recognizing defect patterns of cold mill strip," in Proceedings of the 17th International Conference on Industrial and Engineering Applications of Artificial Intelligence and Export Systems (IEA/AIE 2004), pp. 341-350, Ottawa, ON, Canada, May 2004.

[15] X. Wang, J. Zhang, N. Masafumi et al., "Efficient phoneme set design using phonetic decision tree in dialogue-based English CALL systems for Japanese students," IEICE Technical Report, vol. 113, no. 366, pp. 47-51, 2013.

[16] A.-K. Koliopoulos, P. Yiapanis, F. Tekiner et al., "A parallel distributed Weka framework for big data mining using spark," in Proceedings of the IEEE International Congress on Big Data: Institute of Electrical and Electronics Engineers, pp. 9-16, New York, NY, USA, July 2015.

[17] C. Chen and J. Linhai, Stand Species Identification Using GF-2 Imagery and Airborne LiDAR Data Based on SVM Classifier, pp. 1-13, IOP Conference Series Earth and Environmental Science, Bristol, UK, 2019.
[18] F. Qiang, L. Yong-Bo, H.-W. Wang et al., “Aero-engine health monitoring method based on E-bayes and DNN fusion decision," in Proceedings of the 2020 2nd International Conference on Advanced Control, Automation and Artificial Intelligence (ACAAI 2020), pp. 97-104, Wuhan, China, January 2020.

[19] Z. Tianjiao, W. Yue, and Y. Wang, "College network forum Bayes classification system: implementation and performance evaluation," in Proceedings of the 12th International Conference on Management of e-Commerce and e-Government (ICMeCG 2018), pp. 391-397, Hong kong, China, June 2018. 